\title{
ON COMPOSITION SERIES IN FINITE GROUPS
}

\author{
STEVEN BAUMAN
}

\begin{abstract}
TheORem. Let $G$ be a finite group and $H$ a solvable subgroup of $G$. Suppose that the Schreier conjecture holds. Then $G$ is solvable iff $G$ has an $H$-composition series.
\end{abstract}

Let $G$ be a group and $H \leqq G$. Let $\left\{G_{i}\right\}_{0}^{n}$ be subnormal series with $G_{n}=\langle 1\rangle$ and $G_{0}=G$. This series is called an $H$-composition series if $H$ normalizes each $G_{i}$ and if there exists no subgroup $X$ properly between $G_{i+1}$ and $G_{i}$ which is normalized by $H$.

If $G$ is a finite solvable group then for all $H \leqq G$ such $H$-composition series exist. These can be obtained by refinement into irreducible $H$-factors of any chief series of $G$. If $G$ is not solvable then, for particular $H$, such series may not exist. This is easily seen by letting $G$ be simple nonabelian and $H$ any proper subgroup.

The object of this note will be to shed some light on restrictions that one must have on finite groups $G$ and $H \leqq G$ if such $H$-composition series occur. All groups are finite. If $\left\{G_{i}\right\}_{0}^{n}$ is a subnormal series of $G$ we denote by $G^{(i)}$ the factor $G_{i-1} / G_{i}$ and call $\left\{G^{(i)}\right\}_{1}^{n}$ the factors of the series. A factor of $G$ is a group $R / S$ where $S \unlhd R \leqq G$. If $K / L$ is a factor of $G$ then we can in a natural way define $\operatorname{Aut}_{G}(K / L)$ as $N(K) \cap N(L) / C(K / L)$ and $\operatorname{Out}_{G}(K / L)$ as $N(K) \cap N(L) / K C(K / L)$. These groups correspond to the automorphisms and outer automorphisms that $G$ induces on the factor $K / L$. If $\Sigma$ is a group, then $\Sigma$ is said to be involved in $G$ if $\Sigma$ is isomorphic to some factor of $G$. If $\Sigma$ is a nonabelian simple group, then $K / L$ is called a $\Sigma$-factor if it is the direct product of isomorphic copies of $\Sigma$.

If $\Sigma$ is a nonabelian simple group the Schreier conjecture states that $\operatorname{Out}(\Sigma)=\operatorname{Aut}(\Sigma) / \operatorname{In}(\Sigma)$ is a solvable group. In what follows, if $K / L$ is a simple nonabelian factor of $G$ then if $\operatorname{Out}_{G}(K / L)$ is solvable we will say that $G$ satisfies the Schreier conjecture with respect to the factor $K / L$. Our result is

THEOREM. Let $H<G$ with $H$-composition series $\left\{G_{i}\right\}_{0}^{n}$. Let $\Sigma$ be a nonabelian simple group and $G^{(i)}$ be a $\Sigma$-factor. Suppose $G$ satisfies the $S c h r e i e r$

Received by the editors May 30, 1972.

AMS (MOS) subject classifications (1970). Primary 20D30.

(c) American Mathematical Society 1973 
conjecture with respect to the simple summands of $G^{(i)}$. Then $\Sigma$ is involved in $\mathrm{H}$.

(Note that the simple summands of $G^{(i)}$ are all conjugate by elements of $H$ and thus induced automorphism groups are isomorphic.)

LemMA 1. Let $G$ be a semidirect product of $K$ by $H$. If $H$ is maximal in $G$ and solvable then $G$ is solvable.

Proof. By induction on $|G|$ we may assume that $\operatorname{core}_{G}(H)=1$. Let $R / K$ be minimal normal in $G / K$. We have that $R / K$ is a $p$ group and $H=$ $N(R \cap H)$. It follows that $R \cap H \in \operatorname{Syl}_{p}(R)$ since if not we get $R \cap H<$ $N_{R}(R \cap H)$ which together with $R \cap H \triangleleft H$ implies that $R \cap H \triangleleft G$. Let $S \in \operatorname{Syl}_{q}(K)$. The Frattini argument gives that $G=K \cdot N(S)$. Since $(|K|, p)=$ 1 we get, by Sylow's theorem and a suitable choice of $S$, that $R \cap H \leqq$ $N_{R}(S)$. The Frattini argument applied to $R \cap N \leqq N_{R}(S) \triangleleft N(S)$ yields that $N(S)=N_{H}(S) \cdot N_{R}(S)$. Since $R=K \cdot(R \cap H)$, it follows by Dedekind's theorem that $N_{R}(S)=K \cdot(R \cap H) \cdot \cap N(S)=(R \cap H) \cdot N_{K}(S)$. Thus we have that $N(S)=N_{H}(S) \cdot N_{K}(S)$ or that $G=N_{H}(S) \cdot K$. Since $G=H K$, $H \cap K=1$, we arrive at $N_{H}(S)=H$ or $H<N(S)$. This forces $K=S$ and thus $G$ is solvable.

Lemma 2. Let $G$ be a semidirect product of $K$ by $H$ with $H$ maximal in $G$. Suppose $K$ is a $\Sigma$-factor where $\Sigma$ is a nonabelian simple group. If $G$ satisfies the Schreier conjecture for any simple direct summand of $K$ then $\Sigma$ is involved in $H$.

Proof. Let $S$ be a simple direct summand of $K$. Then $S$ is isomorphic to $\Sigma$. We can choose $h_{1}, \cdots, h_{t}$ a full set of coset representatives of $N_{H}(S)$ in $H$ and $K=S^{h_{1}} \times \cdots \times S^{h_{t}}$. Suppose a $1<R \leqq S$ such that $N_{H}(S)$ normalizes $R$. Since for $x \in N_{H}(S), \exists 1 \leqq l \leqq k, y \in N_{H}(S)$, such that $h_{i} \times h_{i}=y \cdot h_{l}$ we get that $R^{h_{1}} \times \cdots \times R^{h_{t}}$ is normalized by $H$. This yields that $R=S$. Now induction applies to the semidirect product of $S$ by $N_{H}(S)$. If $\left|S \cdot N_{H}(S)\right|<|G|$ we conclude that $\Sigma$ is involved in $N_{H}(S)$ and therefore in $H$. Thus we can conclude that $K=S$. Let $T=C(S)$. Then $T \triangleleft G$ and $T \cap$ $S=1$. If $T \nless H$ since $H$ is maximal we get that $G=H T$. It follows that $S \cong$ $S T / T \cong S T \cap H / T \cap H$ and again $\Sigma$ is involved in $H$. If $T \leqq H$ we look at $G / T$. Our assumption of the Schreier conjecture yields $G / S T$ and thus $H / T$ solvable. Thus Lemma 1 applies to make $G / T$ solvable. This final contradiction, since $S T / T$ is not solvable, proves Lemma 2.

The proof of our theorem follows easily from Lemma 2. By the definition of $H$-composition series it is easy to see that $H$ either covers or avoids each $G^{(i)}$. If $H$ covers this factor then surely $G^{(i)}$ and thus $\Sigma$ is involved in $H$. 
If $H$ avoids $G^{(i)}$ then we are in the situation that $H G_{i-1} / G_{i}$ is a semidirect product of $G_{i-1} / G_{i}$ by $H G_{i} / G_{i}$. By the $H$-irreducibility of $G^{(i)}$ we have that $H G_{i} / G_{i}$ is maximal in $H G_{i-1} / G_{i}$. By our Lemma 2 we are done. Note that $H G_{i-1} / G_{i}$ satisfies the Schreier conjecture with respect to any simple summand of $G^{(i)}$.

Corollary. Let $H \leqq G$ with $H$ solvable. Suppose that $\operatorname{Out}_{a}(\Sigma)$ is solvable for all nonabelian simple factors $\Sigma$ of $G$. Then $G$ is solvable if and only if $G$ has an $H$-composition series.

Department of Mathematics, University of Wisconsin, Madison, Wisconsin 53706 